\title{
Procesos hopping a través del modelo difusional en materiales nanocristalinos usados para aplicaciones fotovoltaicas
}

\author{
A. Dussan ${ }^{1 \bowtie}$, F. Mesa ${ }^{2}$
}

\section{Hopping processes in nanocrystalline materials used for photovoltaic applications using a diffusion model}

\begin{abstract}
Here, we present variable range hopping (VRH) models, nearest neighbor hopping (NNH) and potential barriers present at the grain boundaries, as well as mechanisms of electrical transport predominant in semiconductor materials for photovoltaic applications. We performed dark conductivity measures according to temperature for low temperature regions between 120 and $400 \mathrm{~K}$ in $\mathrm{Si}$ and $\mathrm{Cu}_{3} \mathrm{BiS}_{2}$ and $\mathrm{Cu}_{2} \mathrm{ZnSnSe}_{4}$ compounds. Using the percolation theory, we obtained hopping parameters and the density of states near the Fermi, $N\left(E_{F}\right)$ level for all samples. Using the approach by Mott for VRH, we obtained the diffusion model, which established the relationship between conductivity and density of defect states or localized gap states of the material. The comparative analysis between models evidenced that it is possible to obtain improvement of an order of magnitude in the values of each of the hopping parameters that characterize the material.
\end{abstract}

Keywords: Semiconductors; hopping transport; diffusion model.

Edited by Beynor Paez $₫$ \& Alberto Acosta

1 Departamento de Física, Grupo de Materiales Nanoestructurados, Universidad Nacional de Colombia - Bogotá, Colombia.

2 Unidad de Estudios Universitarios, Universidad del Rosario, Bogotá, Colombia.

Received: 22-11-2013 Accepted: 21-03-2014

Published on line: 14-04-2014

Citation: Dussan A, Mesa F (2014) Procesos Hopping a través del modelo difusional en materiales nanocristalinos usados para aplicaciones fotovoltaicas. Universitas Scientiarum 19(2): 107-113 doi: 10.11144/Javeriana.SC19-2.phmd

Funding: Universidad Nacional de Colombia - DIB

Electronic supplementary material: N/A

\section{Introducción}

Materiales micro, nano y policristalinos han suscitado un especial interés durante los últimos años, debido a sus potenciales aplicaciones en dispositivos optoelectrónicos, tales como, sensores transistores y celdas solares fotovoltaicas (Chen et al. 2011, Chen et al. 2012, Kang et al. 2012, Miller et al. 2012, Kim et al. 2013). Es de anotar, que el estudio y comprensión, tanto teórico como experimental, de los mecanismos de transporte de carga que gobiernan a este tipo de materiales, continúan siendo poco claros y dependen, en general, de la densidad de estados (DOS), la posición del nivel de Fermi y el campo aplicado, entre otros (Dantus et al. 2011, Shen et al. 2013). 
Para mayor comprensión de los procesos involucrados en este aspecto, se han realizado numerosos trabajos que correlacionan las propiedades ópticas, eléctricas y de microestructura, con las propiedades de transporte en materiales nanocristalinos basados en películas delgadas, entre los que se encuentran: $\mu \mathrm{c}-\mathrm{Si}: \mathrm{H}, \mathrm{CuInTe}_{2}, \mathrm{CdS}_{1-\mathrm{x}} \mathrm{Se}_{\mathrm{x}}$, $\mathrm{Cu}_{3} \mathrm{BiS}_{2}$ (Dussan et al. 2008, Mesa et al. 2009, Yadav et al. 2010). Se ha reportado que para la región de bajas temperaturas, bien abajo de la temperatura ambiente $(\mathrm{T}<300 \mathrm{~K})$, la conductividad a oscuras no exhibe una dependencia térmicamente activada con la temperatura (Arredondo et al. 2010, Huang et al. 2013), la cual es un caso común para la región de altas temperaturas. Uno de los procesos que puede ser considerado para el transporte de portadores en esta región, es el de tunelamiento desde los estados ocupados a los estados desocupados involucrando uno o más fotones; este proceso es denominado "hopping" (Dalvi et al. 2012, Eginligil et al. 2012).

En este trabajo se realizaron medidas de conductividad en oscuro $\left(\sigma_{\text {osc }}\right)$ en función de la temperatura para películas delgadas nanocristalinas de Si y compuestos $\mathrm{Cu}_{3} \mathrm{BiS}_{2}$ y $\mathrm{Cu}_{2} \mathrm{ZnSnSe}_{4}$. Se presenta el modelo denominado "Modelo Difusional" que tiene en cuenta la relación entre el coeficiente de difusión y la probabilidad de hopping; para el cálculo de los párametros que caracterizan el transporte VRH correlacionandolo con los datos obtenidos por la teoría de percolación.

Para el caso de tres dimensiones los autores (Mott 1969, Morthekai et al. 2012) establecieron que, si la densidad de estados (DOS) es constante en un rango de energía $\mathrm{k}_{\mathrm{B}} \mathrm{T}$ alrededor del nivel de Fermi, la conductividad a oscuras en función de la temperatura puede ser expresada como:

$$
\sigma_{o s c}=\sigma_{0} \exp \left[-\left(\frac{T_{0}}{T}\right)^{1 / 4}\right]
$$

donde: $\sigma_{\text {osc }}$ es la conductividad a oscuras, $\mathrm{T}$ es la temperatura y $\mathrm{T}_{0}, \sigma_{0}$ son constantes que dependen del material.

A medida que la temperatura $T$ tiende a ser mucho más pequeña tanto el número como la energía de los fonones decrece de modo que la probabilidad de que un electrón salte energéticamente de un estado a otro vía proceso asistido por fonón, llega a ser menos favorable. En este caso, los portadores muestran una tendencia a realizar saltos a más grandes distancias para encontrar sitios que energéticamente están más cerca que sus vecinos próximos. Este mecanismo, es el que se conoce con el nombre de hopping de Rango Variable. Usando la teoría clásica de percolación la temperatura característica de Mott en el caso tridimensional puede ser estimada a partir de la expresión:

$$
T_{0}=\frac{\mathrm{C}^{4} \alpha^{3}}{k_{B} N_{F}}
$$

donde: $\mathrm{C}$ y $\alpha^{-1}$ son parámetros que varían de acuerdo a los materiales en consideración. Otros parámetros que caracterizan el transporte vía VRH corresponden a la energía de activación hopping $\mathrm{W}_{\text {hop }}$ y el rango hopping $R_{\text {hop }}$.

$W_{b o p}=k_{B}\left(T_{0} T^{3}\right)^{1 / 4}$ y $R_{b o p}=\frac{3}{8} T_{0}^{1 / 4} T_{0}^{-1 / 4} \frac{1}{\alpha}$

En orden de mejorar los cálculos realizados tanto para $\mathrm{R}_{\text {hop }}$ como para $\mathrm{W}_{\text {hop }}$ obtenidos por medio de la teoría de percolación, el modelo difusional (Dussan et al. 2005) desde un punto de vista fenomenológico considera que el portador hopping es difusivo con un coeficiente de difusión dado por:

$\mathrm{D}_{\text {hop }}=\frac{1}{6} \mathrm{R}^{2} \mathrm{P}_{\text {hop }}$ con $P_{\text {bop }}=v_{p h} \exp \left(-2 \alpha \mathrm{R}-W / k_{B} T\right)$

donde: R es el rango o distancia hopping promedio, $\mathrm{P}_{\text {hop }}$ es la probabilidad de transición hopping entre dos estados separados por una distancia $R, \nu_{\text {ph }}$ es la frecuencia del fonón asociada con los procesos hopping, exp $(-2 \alpha \mathrm{R})$ es el factor de tunneling determinado por el ovelarping de las funciones de onda, $\exp \left(-\mathrm{W} / \mathrm{k}_{\mathrm{B}} \mathrm{T}\right)$ es el factor de Boltzmann que relaciona la energía $\mathrm{W}$ entre los estados inicial y final de los procesos hopping y $\alpha$ es la longitud de localización. 
A partir de consideraciones energéticas y valores adecuados para los parámetros numéricos asociados a cada uno de los materiales, se pueden reescribir las expresiones para $\mathrm{R}_{\text {hop }}$ como para $\mathrm{W}_{\text {hop }}$ que difieren en un factor numérico conteniendo una dependencia con el parámetro $\mathrm{C}$ introducido en la temperatura característica de Mott para el caso 3D en la teoría de percolación (Ecuación 5). Con este nuevo factor para cada una de las expresiones, se corrigen los resultados obtenidos a partir de la teoría de percolación y se obtiene una correspondencia con los reportados por el modelo difusional.

$$
\mathrm{W}_{d i f}=\frac{\mathrm{A}}{\mathrm{C}^{2}} k_{B}\left(\mathrm{~T}_{0} \mathrm{~T}^{3}\right)^{1 / 4} \mathrm{y}_{d i f}=\frac{\mathrm{B}}{\mathrm{C}} \mathrm{T}_{0}^{1 / 4} \mathrm{~T}^{-1 / 4} \frac{1}{\alpha}
$$

donde: $\mathrm{A}=(128 / 9 \pi)$ y $\mathrm{B}=(9 / 8 \pi) 1 / 4$

\section{Materiales y métodos}

Se realizaron medidas de conductividad eléctrica en función de la temperatura a muestras semiconductoras de compuestos $\mu \mathrm{cSi}, \mathrm{Cu}_{3} \mathrm{BiS}_{2}$ y $\mathrm{Cu}_{2} \mathrm{ZnSnSe}_{4}$. Las medidas se realizaron usando el método de Van Der Pauw (de acuerdo a la norma ASTM F76) en un sistema de vacío, variando la temperatura entre 120 y $420 \mathrm{~K}$. Se depositaron contactos de Al para todas las muestras con una disposición tipo coplanar. Los parámetros de depósito de la muestras pueden ser revisados a partir de los reportes realizados con cada uno de los materiales (Dussan et al. 2009).

Basados en el formalismo clásico de la teoría de percolación se calcularon la densidad de estados cerca del nivel de Fermi, $\mathrm{N}\left(\mathrm{E}_{\mathrm{F}}\right)$, y los parámetros de hopping que soportan el modelo de hopping de Rango Variable (VRH).

\section{Resultados}

Las medidas de conductividad en oscuro en función de 1000/T para el conjunto de muestras nanocristalinas de $\mu \mathrm{cSi}: \mathrm{H}$ (B), $\mathrm{Cu}_{3} \mathrm{BiS}_{2}$ y compuesto cuaternario $\mathrm{Cu}_{2} \mathrm{ZnSnSe}_{4}$ fueron realizadas en un amplio rango de temperaturas (Figura 1). Las condiciones de preparación de las muestras, esto es, $\mu \mathrm{cSi}: \mathrm{H}$ compensado con $50 \mathrm{ppm}$ de boro, $\mathrm{Cu}_{3} \mathrm{BiS}_{2}$ depositado

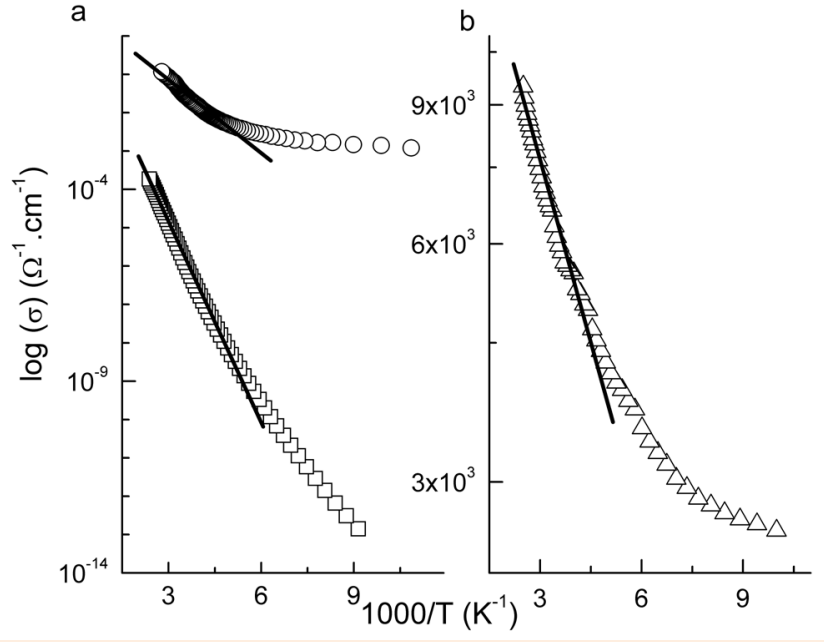

Fig. 1. Medidas de conductividad en función de la temperatura para muestras nanocristalinas: a. $\mu \mathrm{cSi}: \mathrm{H}$ (B) $(\square \mathrm{Ea}=0,37 \mathrm{eV}), \mathrm{Cu}_{3} \mathrm{BiS}_{2}(\square \mathrm{Ea}=0.14$ $\mathrm{eV})$, b. Compuesto cuaternario $\mathrm{Cu}_{2} \mathrm{ZnSnSe}_{4}(\Delta$ $\mathrm{Ea}=0,33 \mathrm{eV})$. Se indica la línea recta que sigue la ecuación 1 y se ajusta la región de altas temperaturas para el cálculo de la Ea en cada caso.

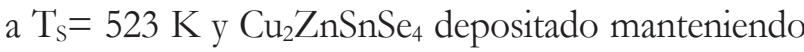
la $T_{\mathrm{S}(\mathrm{Cu})}$ en $673 \mathrm{~K}$, han sido reportadas anteriormente (Dussan et al. 2009, Dussan et al. 2012).

El comportamiento de conductividad (Figura 2) para la región de bajas temperaturas (descrito a partir de Ecuación 1), se muestra en gráfico de logaritmo de conductividad en función de $T^{1 / 4}$ para las muestras nanocristalinas.

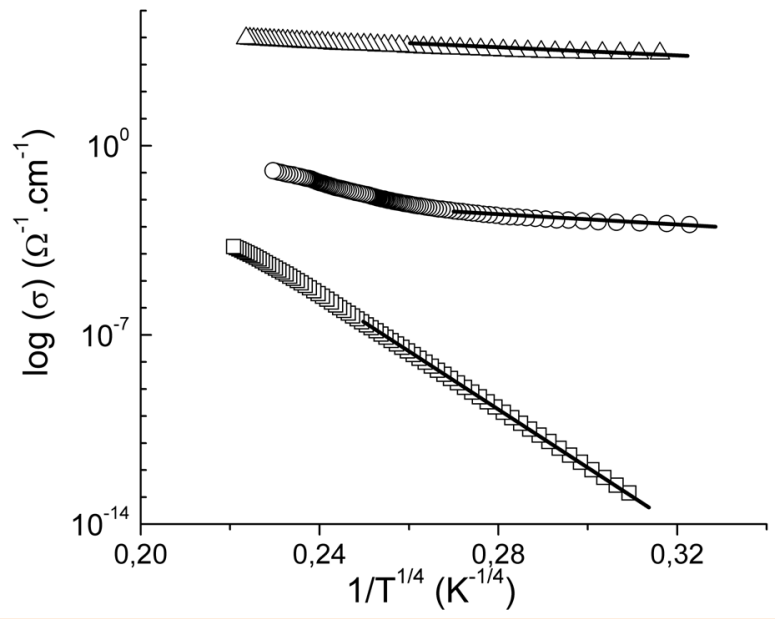

Fig. 2. Conductividad en función de $\mathrm{T}^{1 / 4}$ para muestras de a. $\mu \mathrm{cSi}: \mathrm{H}$ (B) ( $\square), \mathrm{Cu} 3 \mathrm{BiS} 2(\mathrm{O}), \mathbf{b}$. Compuesto cuaternario $\mathrm{Cu}_{2} \mathrm{ZnSnSe}_{4}(\Delta)$. La línea recta representa el ajuste lineal encontrado para la región de bajas temperaturas. 
Los procesos hopping que dan evidencia del los mecanismos de transporte para la región de bajas temperaturas pueden ser asociados tanto a saltos entre vecinos cercanos (NNH) como saltos de rango variable (VRH). En forma esquemática se puede representar el camino que describen los portadores a través de los distintos mecanismos de hopping: VRH y NNH (Figura 3).

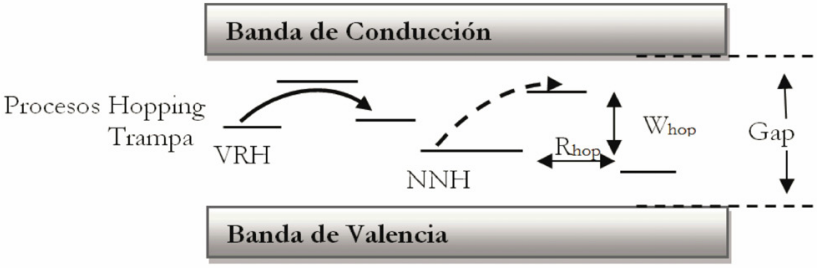

Fig. 3. Esquema de los procesos hopping VRH y NNH que dan cuenta del transporte eléctrico en materiales semiconductores para la región de baja temperatura. $R_{\text {hop }} y W_{\text {hop }}$ indican el rango y la energía de activación hopping.

Se reportan, a partir de la teoría de percolación (Tabla 1) y lo planteado por el modelo difusional (Ecuaciones 3-5), los parámetros que caracterizan el transporte VRH. Un análisis comparativo entre ambos modelos permitió evidenciar, que a partir del modelo difusional es posible obtener una mejora significativa en los valores obtenidos para cada uno de los parámetros hopping que caracterizan estos en el rango de $\mathrm{T}>300 \mathrm{~K}$ que se ajusta a la expresión de Arrhenius (Ecuación 6) presentando una única energía de activación, Ea, en cada una las muestras (Figura 1). Los valores de la Ea para cada material son reportados.

$$
\sigma_{o s c}=\sigma_{0} \exp \left[-\left(\frac{E_{\mathrm{a}} \alpha^{3}}{K_{B} T}\right)\right]
$$

De manera general se observa que los materiales con presencia de $\mathrm{Cu}$ en su composición exhiben una conductividad mayor a lo encontrado para el caso del Si. Esto, está relacionado directamente con la contribución de los átomos de $\mathrm{Cu}$ en el proceso de conducción de portadores en la red, tanto del $\mathrm{Cu}_{3} \mathrm{BiS}_{2}$, como del compuesto cuaternario $\mathrm{Cu}_{2} \mathrm{ZnSnSe}_{4}$ (Figura 1). Sin embargo, el interés se centra en el estudio de los posibles mecanismos de transporte para la región de bajas temperaturas, donde el ajuste lineal no es posible.

Por otra parte, se ha incluido de manera adicional una de las medidas realizadas al silicio microcristalino teniendo en cuenta que este material es también uno de los semiconductores más usados en la fabricación de dispositivos fotovoltaicos (Figura 1). Se puede observar que los ajustes realizados a las regiones de bajas temperaturas sugieren que los procesos electrónicos dominantes como mecanismos de conducción ocurren en una banda de energía más angosta alrededor del nivel de Fermi, a través de estados localizados.

Tabla 1. Valores obtenidos de los parámetros hopping para muestras de $\mu \mathrm{cSi}: \mathrm{H}$ (B), $\mathrm{Cu}_{3} \mathrm{BiS}_{2}$ y compuesto cuaternario $\mathrm{Cu}_{2} \mathrm{ZnSnSe}_{4}$. El parámetro de localización considerado fue de $10 \AA$ para el $\mu \mathrm{cSi}: \mathrm{H}$ (B) y $257 \AA$ para los compuestos $\mathrm{Cu}_{3} \mathrm{BiS}_{2}$ y $\mathrm{Cu}_{2} \mathrm{ZnSnSe}_{4}$ (Thamilselvan et al. 2003, Dussan et al. 2005).

\section{T. Percolación}

M. Difusional

\begin{tabular}{cccccc} 
Muestra & $\mathbf{N}\left(\mathbf{E}_{\mathrm{F}}\right)\left(\mathbf{c m}^{-3} \mathbf{e V}^{-1}\right)$ & $\mathbf{R}_{\text {hop }}(\mathbf{c m})$ & $\mathbf{W}_{\text {hop }}(\mathbf{e V})$ & $\mathbf{R}_{\text {hop }}(\mathbf{c m})$ & $\mathbf{W}_{\text {hop }}(\mathbf{e V})$ \\
\hline $\mathrm{Si}$ & $2.139 \times 10^{16}$ & $1.46744 \times 10^{-6}$ & 0.36128 & $1.46956 \times 10^{-6}$ & 0.09628 \\
$\mathrm{Cu}_{3} \mathrm{BiS}_{3}$ & $3.269 \times 10^{17}$ & $3.83624 \times 10^{-6}$ & 0.05145 & $3.84156 \times 10^{-6}$ & 0.01293 \\
$\mathrm{Cu}_{2} \mathrm{ZnSnSe}_{4}$ & $3,403 \times 10^{18}$ & $1,25004 \times 10^{-7}$ & 0.02052 & $1,03322 \times 10^{-7}$ & 0.01359
\end{tabular}

\section{Discusión}

A partir de las medidas de conductividad se puede observar comportamiento lineal para las temperaturas
Este mecanismo, el cual es conocido como conducción hopping puede evidenciarse como hopping de vecinos cercanos (NNH: nearest neighbor hopping) o de rango variable, VRH (Koval et al. 2011, Lisunov et 
al. 2013). Para las muestras consideradas se observa que las curvas pueden ser ajustadas con una función lineal seguida por la Ecuación 1; este comportamiento evidencia el transporte de portadores de carga vía procesos hopping entre estados presentes en el gap del material y la banda de valencia o conducción. Un diagrama esquemático de los procesos hopping VRH y $\mathrm{NNH}$ en un material semiconductor pueden ser explicados a través de los procesos que ocurren vía movimiento de los portadores (Figura 3).

Los resultados correspondientes al rango hopping tanto por el modelo difusional como por medio de la teoría de percolación resultaron ser los mismos (Tabla 1). Sin embargo, la energía de activación hopping $W_{\text {hop }}$ calculada a partir de la teoría de percolación es notoriamente mucho mayor a la obtenida por medio del modelo difusional; este hecho implica que el valor de $W_{\text {hop }}$, a partir del modelo difusional, ratifica la suposición inicial de que el ancho de banda, alrededor de $\mathrm{E}_{\mathrm{Fermi}}$, la cual es responsable de la conducción para el mecanismo de VHR, es muy angosto para bajas temperaturas. En concordancia con lo anterior se puede observar que los valores de $\mathrm{R}$ aumentan a medida que la temperatura disminuye mientras lo contrario ocurre con la energía de activación hopping (Figura 4). Este hecho caracteriza el mecanismo de

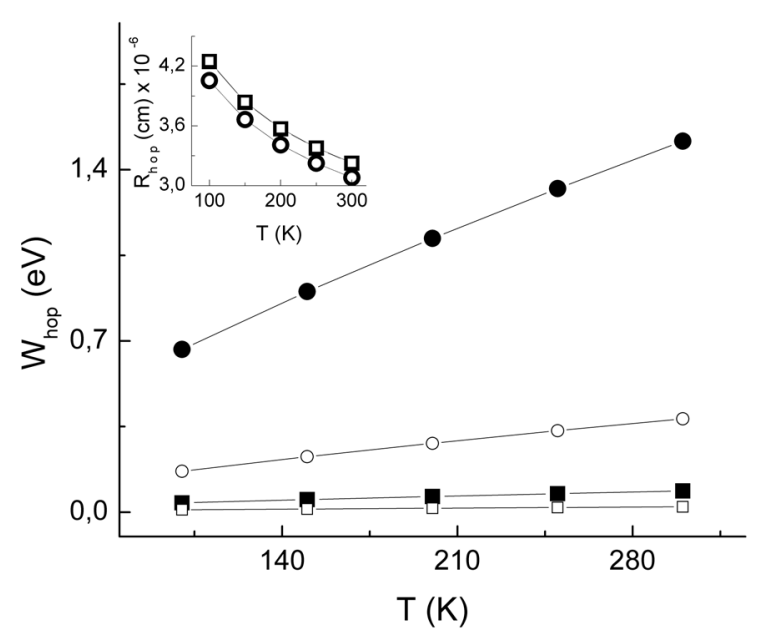

Fig. 4. Variación de los parámetros $R_{\text {hop }} y W_{\text {hop }}$ en función de la temperatura para muestras nanocristalinas de de $\mu \mathrm{cSi} \mathrm{H}$ (B), $\mathrm{Cu}_{3} \mathrm{BiS}_{2}$. Los símbolos oscuros representan los valores obtenidos a partir de la Teoría de percolación y los símbolos abiertos al modelo difusional.
VHR, donde los portadores tenderían a realizar saltos a más grandes distancias en orden a encontrar sitios que permanezcan energéticamente más cerca que sus vecinos cercanos (Misra et al. 2010, Serin et al. 2011, Li et al. 2012, Phan et al. 2012).

\section{Conclusión}

En este trabajo se realizaron medidas de conductividad a oscuras en función de la temperatura a muestras de $\mathrm{Si}$ y compuestos $\mathrm{Cu}_{3} \mathrm{BiS}_{2}$ y $\mathrm{Cu}_{2} \mathrm{ZnSnSe}_{4}$. A partir de la teoría de percolación y el modelo difusional se obtuvieron los parámetros hopping $\mathrm{R}_{\text {hop }} \mathrm{y} \mathrm{W}_{\text {hop }}$, así como también la densidad de estados cerca del nivel de Fermi, $\mathrm{N}\left(\mathrm{E}_{\mathrm{F}}\right)$, para todas las muestras. A partir del modelo difusional fue posible obtener hasta en un orden de magnitud la diferencia de la energía de activación entre ambos modelos. Los valores correspondientes al rango hopping son reproducidos y están en concordancia con los obtenidos a través de la teoría de percolación. Otros materiales podrían ser ajustados a través de estos modelos y obtener los parámetros que caracterizan el transporte.

\section{Agradecimientos}

Este trabajo fue soportado por proyectos de la Universidad Nacional de Colombia - DIB.

\section{Conflicto de intereses}

Este trabajo no presenta conflicto de intereses.

\section{Referencias}

Arredondo CA, Gordillo G (2010) Photoconductive and electrical transport properties of AgInSe2 thin films prepared by co-evaporation. Physica B: Condensed Matter 405:3694-3699 doi: 10.1016/j.physb.2010.05.068

Chen T, Huang Y, Dasgupta A, Luysberg M, Houben L et al. (2012) Microcrystalline silicon carbide window layers in thin film silicon solar cells. Solar Energy Materials and Solar Cells 98:370-378 doi: 10.1016/j.solmat.2011.11.039

Chen X, Lin Q, Ni J, Zhang D, Sun J et al. (2011) Textured surface boron-doped $\mathrm{ZnO}$ transparent conductive oxides on polyethylene terephthalate substrates for Si-based thin film solar cells. Thin Solid Films 520:12631267 doi: 10.1016/j.tsf.2011.04.199

Dalvi A, Reddy NP, Agarwal SC (2012) The Meyer-Neldel rule and hopping conduction. Solid State Communications 152:612-615 doi: 10.1016/j.ssc.2012.01.018 
Dantus C, Rusu RS, Rusu GI (2011) On the mechanism of electronic transport in polycrystalline $\mathrm{CdO}$ thin films. Superlattices and Microstructures 50:303-310 doi: 10.1016/j. spmi.2011.07.008

Dussan A, Buitrago RH (2005) Transport mechanism in lightly doped hydrogenated microcrystalline silicon thin films. Journal of Applied Physics 97:043711 doi: $10.1063 / 1.1848193$

Dussan A, Buitrago RH, Koropecki RR (2008) Microcrystalline silicon thin films: A review of physical properties. Microelectronics Journal 39:1292-1295 doi: 10.1016/j.mejo.2008.01.019

Dussan A, Mesa F, Botero M, Gordillo G (2009) Electrical and optical properties of thin films with a SnS2 Bi2S3 alloy grown by sulphurization. Journal of Physics: Conference Series 167:012018 doi: 10.1088/17426596/167/1/012018

Dussan A, Mesa F, Botero M, Gordillo G (2012) Electrical and optical properties of thin films with a SnS2 -Bi2S3 alloy grown by sulphurization. Journal of Physics: Conference Series 167:012018 doi: 10.1088/17426596/167/1/012018

Eginligil M, Zhang W, Kalitsov A, Lu X, Yang H (2012) Tunneling behavior of bismuth telluride nanoplates in electrical transport. Chemical Physics Letters 546:125-128 doi: 10.1016/j.cplett.2012.07.068

Huang X, Wu C, Lu H, Ren F, Chen D et al. (2013) Temperature and gate bias dependence of carrier transport mechanisms in amorphous indium-galliumzinc oxide thin film transistors. Solid-State Electronics 86:41-44 doi: 10.1016/j.sse.2013.04.025

Kang DW, Kwon JY, Shim J, Lee HM, Han MK (2012) Highly conductive $\mathrm{GaN}$ anti-reflection layer at transparent conducting oxide/Si interface for silicon thin film solar cells. Solar Energy Materials and Solar Cells 105:317-321 doi: 10.1016/j.solmat.2012.06.041

Kim SJ, Gunduz B, Yoon DH, Kim HJ, Al-Ghamdic A et al. (2013) Photofield effect and photoresponse properties of the transparent oxide-based BaInZnO thin-film transistors. Sensors and Actuators A: Physical 193:1-12 doi: 10.1016/j.sna.2013.01.002

Koval Y, Lazareva I, Müller P (2011) Coulomb gap variable range hopping in graphitized polymer surfaces. Synthetic Metals161:528-534 doi:10.1016/j.synthmet.2011.01.007

Li L, Chung KS, Jang J (2012) Carrier concentration dependentbimolecular recombination coefficient model in two dimensional hopping system. Synthetic Metals 162:702-704 doi: 10.1016/j.synthmet.2012.02.013

Lisunov KG, Guk M, Nateprov A, Levcenko S, Tezlevan V (2013) Features of the acceptor band and properties of localized carriers from studies of the variable-range hopping conduction in single crystals of $\mathrm{p}-\mathrm{Cu} 2 \mathrm{ZnSnS4}$.
Solar Energy Materials and Solar Cells 112:127-133 doi: 10.1016/j.solmat.2013.01.02

Mesa F, Dussan A, Gordillo G (2009) Evidence of trapping levels and photoelectric properties of $\mathrm{Cu}_{3} \mathrm{BiS}_{2}$ thin films. Physica B: Condensed Matter 404:5227-5230 doi: 10.1016/j.physb.2009.08.302

Miller JB, Ashok T, Lee S, Broitmanet E (2012) Zinc oxidebased thin film functional layers for chemiresistive sensors. Thin Solid Films 520:6669-6676 doi: 10.1016/j. tsf.2012.07.016

Misra SK, Andronenkoa SI, Asthana S, Bahadur D (2010) A variable temperature EPR study of the manganites (La1/3Sm2/3) 2/3SrxBa0.33-xMnO3 ( $\mathrm{x}=0.0,0.1,0.2$, 0.33): Small polaronhopping conductivity and Griffiths phase. Journal of Magnetism and Magnetic Materials 322:2902-2907 doi: 10.1016/j.jmmm.2010.05.003

Mott NF (1969) Philos. Mag 19:835

Morthekai P, Thomas J, Pandian MS, Balaram V, Singhvi AK (2012) Variable range hopping mechanism in bandtail states of feldspars: A time-resolved IRSL study. Radiation Measurements 47:857-863

Phan BT, Choic T, Romanenko A, Lee J (2012) Hopping and trap controlled conduction in $\mathrm{Cr}$-doped $\mathrm{SrTiO} 3$ thin films. Solid-State Electronics 75:43-47 doi: 10.1016/j. sse.2012.05.007

Serin N, Yildiz A, Alsaç AA, Serinet T (2011) Estimation of compensation ratio by identifying the presence of different hopping conduction mechanisms in $\mathrm{SnO} 2$ thin films. Thin Solid Films 519:2302-2307 doi: 10.1016/j. tsf.20

Shen HP, Zhao CY (2013) Analytical considerations of light transport in nanostructured homogeneous/ inhomogeneous thin films. Thin Solid Films 542:204-209 doi: 10.1016/j.tsf.2013.06.066

Thamilselvan M, Premnazeer K, Mangalaraj D, Narayandass SK (2003) Field and temperature-dependent electronic transport parameters of amorphous and polycrystalline GaSe thin films. Physica B: Condensed Matter 337:404-412 doi: 10.1016/S0921-4526(03)00444-7

Yadav AA, Masumdar EU (2010) Optical and electrical transport properties of spray deposited $\mathrm{CdS}_{1-\mathrm{x}} \mathrm{Se}_{\mathrm{x}}$ thin films. Journal of Alloys and Compounds 505:787-792 doi: 10.1016/j.jallcom.2010.06.141 
Procesos Hopping a través del modelo difusional en materiales nanocristalinos usados para aplicaciones fotovoltaicas

Resumen. Se presentan los modelos de hopping de rango variable (variable range hopping; VRH), vecinos cercanos (nearest neighbor hopping; NNH) y barreras de potencial presentes en las fronteras de grano; como mecanismos de transporte eléctrico predominantes en los materiales semiconductores para aplicaciones fotovoltaicas. Las medidas de conductividad a oscuras en función de temperatura fueron realizadas para región de bajas temperaturas entre 120 y $400 \mathrm{~K}$ con $\mathrm{Si}$ y compuestos $\mathrm{Cu}_{3} \mathrm{BiS}_{2}$ y $\mathrm{Cu}_{2} \mathrm{ZnSnSe}_{4}$. Siguiendo la teoría de percolación, se obtuvieron parámetros hopping y la densidad de estados cerca del nivel de Fermi, $\mathrm{N}\left(\mathrm{E}_{\mathrm{F}}\right)$, para todas las muestras. A partir de los planteamientos dados por Mott para VRH, se presentó el modelo difusional, que permitió establecer la relación entre la conductividad y la densidad de estados de defecto o estados localizados en el gap del material. El análisis comparativo entre modelos, evidenció, que es posible obtener mejora hasta de un orden de magnitud en valores para cada uno de los parámetros hopping que caracterizan el material.

Palabras clave: Semiconductores; transporte hopping; modelo difusional.
Processos Hopping através do modelo de difusáo em materiais nanocristalinos utilizados para aplicaçóes fotovoltaicas

Resumo. Apresentam-se os modelos de hopping de categoria variável (variable range hopping; VRH), vizinhos próximos (nearest neighbor hopping, NNH) e barreiras de potenciais presentes nas fronteiras de grãos; como mecanismo de transporte elétrico predominantes nos materiais semicondutores para aplicações fotovoltaicas. As medidas de condutividade no escuro em função da temperatura foram realizadas para região de baixas temperaturas entre 120 e $400 \mathrm{~K}$ com Si e compostos $\mathrm{Cu}_{3} \mathrm{BiS}_{2}$ e $\mathrm{Cu}_{2} \mathrm{ZnSnSe}_{4}$. Seguindo a teoria da percolação obtiveram-se parâmetros hopping e a densidade de estados próximos do nível Fermi[BO1] $\mathrm{N}\left(\mathrm{E}_{\mathrm{F}}\right)$ para toda a amostra. A partir das abordagens seguidas por Mott para VRH, apresentou-se o modelo de difusão, que permitiu estabelecer a relação entre a condutividade e a densidade de estados de defeito ou estados localizados no gap do material. A análise comparativa dos modelos mostrou que é possível obter melhoria até de uma amplitude de magnitude em valores para cada um dos parâmetros hopping que caracterizam o material.

Palavras-chave: Semicondutores; transporte hopping; modelo de difusão. 